16), mean body mass index was $33 \mathrm{~kg} / \mathrm{m}^{2}$ (SD, 10 ), $73 \%$ were male; $67 \%$ were white Americans, $25 \%$ were African Americans and $7 \%$ other/mixed race/ethnicity. Participants took a median of 35 minutes to complete study assessment.

Almost half of the participants were taking medications for the treatment of gout: allopurinol, $42 \%$; febuxostat, $1 \%$, probenecid, $0 \%$; colchicine, $29 \%$. Forty-five percent participant were taking none of these medications. $41 \%$ smoked ever, $27 \%$ were using a special diet and participants had alcohol use an average of 2 days in the last week. Average number of gout flares were four in the last year. Dietary assessments showed that average daily intakes were as follows: calories, 2005; carbohydrate, $221 \mathrm{gm}$; fat, $82 \mathrm{gm}$; fiber, $19 \mathrm{gm}$; caffeine, $197 \mathrm{ml}$. The HEI2010 score of 64 was comparable to what was observed with NHANES for people in the average age range of this study.

Conclusions: Patients recruited in an Internet gout study, successfully responded to assessments, and had patient characteristics similar to gout populations described previously. The dietary assessments in this provide may provide a unique insight to design interventions to improve diet to improve gout outcomes. References:

[1] Singh JA, Bharat A, Edwards NL. An internet survey of common treatments used by patients with gout including cherry extract and juice and other dietary supplements. J Clin Rheumatol. 2015;21(4):225-226. doi:10.1097/RHU.0000000000000246. PubMed PMID: 26010189.

Acknowledgements: This work was supported by a grant from UAB COERE nad UAB MHRC centers.

Disclosure of Interest: J. Singh Grant/research support from: Savient, Takeda, Consultant for: Savient, Takeda, Regeneron, Merz, Iroko, Bioiberica, Crealta and Allergan pharmaceuticals, WebMD, UBM LLC and the American College of Rheumatology, G. MCGWIN: None declared DOI: 10.1136/annrheumdis-2017-eular.2762

\section{AB0880 PHARMACODYNAMIC EFFECTS AND SAFETY OF VERINURAD (RDEA3170) IN COMBINATION WITH ALLOPURINOL VERSUS ALLOPURINOL ALONE IN ADULTS WITH GOUT: A PHASE 2A, OPEN-LABEL STUDY}

R. Fleischmann ${ }^{1}$, P. Winkle ${ }^{2}$, J.N. Miner ${ }^{3}$, X. Yan ${ }^{3}$, L. Hicks ${ }^{3}$, S. Valdez ${ }^{3}$, J. Hall ${ }^{3}$, M. Hernandez-lllas ${ }^{4}{ }^{1}{ }^{1}$ University of Texas, Southwestern Medical Center, Metroplex Clinical Research Center, Dallas, $T X ;{ }^{2}$ Anaheim Clinical Trials, Anaheim, CA; ${ }^{3}$ Ardea Biosciences, Inc., San Diego, CA; ${ }^{4}$ QPS MRA (Miami Clinical Research), Miami, FL, United States

Background: Verinurad (RDEA3170) is a high-affinity, selective URAT1 inhibitor in development for the treatment of gout and asymptomatic hyperuricemia. Objectives: This Phase $2 a$, randomized, open-label, multicenter study investigated the multiple-dose pharmacodynamics (PD), pharmacokinetics (PK), and safety of oral verinurad in combination with allopurinol versus allopurinol alone in adults with gout (NCT02498652).

Methods: Patients aged $\geq 18$ and $\leq 75$ years with gout and serum uric acid $(\mathrm{sUA}) \geq 8 \mathrm{mg} / \mathrm{dL}$ were randomized to 1 of 2 cohorts to receive allopurinol (300 $\mathrm{mg}$ ) in combination with verinurad (dose range $2.5 \mathrm{mg}$ to $20 \mathrm{mg}$ ) and allopurinol $300 \mathrm{mg}$ or $600 \mathrm{mg}$ alone (each treatment period was 7 days). Medications were administered once daily $\sim 30 \mathrm{~min}$ after breakfast (for allopurinol $300 \mathrm{mg}$ b.i.d. group, the second allopurinol dose was in the evening). Colchicine 0.6 $\mathrm{mg}$ for gout flare prophylaxis was initiated at approximately Day -14 (start of urate-lowering therapy [ULT]) washout) or Day -7 if not on ULT. Serial blood and urine samples were measured on Days $-1,1,7,14,21,28$, and 35 for PD and PK endpoints. Safety assessments included adverse events (AEs) and laboratory, electrocardiogram, and vital sign parameters.

Results: Forty-one patients were randomized ( $n=20-21$ per cohort). Serum PD data pooled across cohorts demonstrated maximal \% decrease in sUA from baseline (Emax)at 6-10 h after verinurad and allopurinol combination treatment. Addition of verinurad $(2.5 \mathrm{mg}$ to $20 \mathrm{mg}$ ) to allopurinol decreased sUA in dosedependent manner (Figure). Greater sUA reductions were observed for dose combinations of verinurad $\geq 5 \mathrm{mg}$ with allopurinol $300 \mathrm{mg}$ versus allopurinol 600 $\mathrm{mg}$ alone, while allopurinol $600 \mathrm{mg}$ once daily was equivalent to allopurinol 300

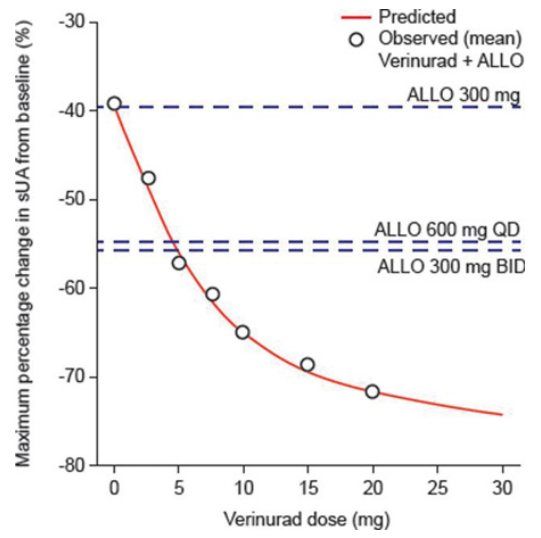

b.i.d. Emax was $46.9 \%, 58.9 \%, 59.9 \%, 67.1 \%, 68.4 \%$, and $74.3 \%$ for verinurad at doses of $2.5,5,7.5,10,15$, and $20 \mathrm{mg}$ in combination with allopurinol $300 \mathrm{mg}$, versus $39.7 \%, 53.8 \%$, and $54.4 \%$ with allopurinol $300 \mathrm{mg}$, allopurinol $600 \mathrm{mg}$, and allopurinol $300 \mathrm{mg}$ b.i.d. alone. No drug-drug interaction on verinurad and allopurinol plasma PK parameters was observed.

Conclusions: Verinurad coadministered with allopurinol dose-dependently decreased SUA. All dose combinations of verinurad and allopurinol in this study were generally well tolerated with no serious AEs or renal-related events during combination treatment.

Disclosure of Interest: R. Fleischmann Grant/research support from: Ardea Biosciences, Inc., P. Winkle Employee of: Anaheim Clinical Trials, J. Miner Employee of: Ardea Biosciences, Inc., X. Yan Employee of: Ardea Biosciences, Inc., L. Hicks Employee of: Ardea Biosciences, Inc., S. Valdez Employee of: Ardea Biosciences, Inc., J. Hall Employee of: Ardea Biosciences, Inc., M. Hernandez-lllas: None declared

DOI: 10.1136/annrheumdis-2017-eular.5429

\section{AB0881 ASSESSMENT OF SUDOMOTOR FUNCTION IN PATIENT WITH GOUT}

J.W. Lee ${ }^{1}$, H.O. Kim ${ }^{2}$, S. Lee ${ }^{3}$, G. Kim ${ }^{4} .{ }^{1}$ Busan St. Mary's Hospital, Busan; ${ }^{2}$ KyungSang University, ChangWon: ${ }^{3}$ Busan National University; ${ }^{4}$ Gosin univeristy, Busan, Korea, Republic Of

Background: Sodorimetry is a non-invasive device measures sweat gland dysfunction using electrochemical skin conductance (ESC) of hands and feet and is useful for assessing peripheral small fiber nerve function. Little is known about the dysfunction of peripheral small fiber nerve in patients with gout.

Objectives: To evaluate the prevalence and characteristics of small fiber neuropath (SFN) in patients with gout compared with a healty control group and to identify factors associated with SFN in gout.

Methods: 80 male patients with well symtom controlled gout (age: $58 \pm 12$ ) and 80 healthy controls were enrolled. Each patient was required to fast over 8 hours before blood samples. Serum fasting glucose, fasting insulin, uric acid, serum 25- $(\mathrm{OH}) \mathrm{D}$, lipid profiles, Creatinine $(\mathrm{Cr})$ and $\mathrm{r}$-GTP were measured. Body mass index (BMI) and Homeostatic model assessment insulin resistance (HOMA IR) were calculated. Patients already diagnosed with hypertension and diabetes were excluded.

Results: The mean feet and hands ESC were significantly lower in the gout group than the control group. Mean Hands ESC was irrelevant to age, BMI, fasting glucose and insulin, HOMA-IR, vit D, uric acid, $\mathrm{Cr}$, and lipids. However, mean feet ESC showed significant correlation with fasting glucose $(r=-0.7, p<0.01)$ and HOMA-IR $(r=-0.25, p=0.03)$.

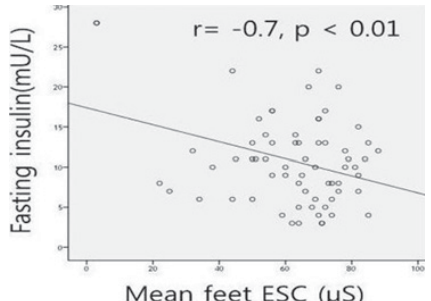

Conclusions: Sudomotor function was significantly lower in patients with gout than the control group. Mean feet ESC was correlated with fasting glucose and insulin resistance in patients with gout. These results suggest that dysfunction of SFN in gout patients is associated with insulin resistance and impaired fasting glucose.

Disclosure of Interest: None declared

DOI: 10.1136/annrheumdis-2017-eular.5789

\section{AB0882 EFFICACY OF INTRALESIONNAL SODIUM THIOSULFATE IN DISABLING TUMORAL CALCINOSIS: ABOUT TWO CASES}

J. Goossens $^{1}$, M. Courbebaisse ${ }^{2}$, V. Ratsimbazafy ${ }^{3}$, D. Bazin ${ }^{4}$, M. Daudon ${ }^{5}$, V. Frochot ${ }^{5}$, P. Richette ${ }^{1}$, F. Lioté ${ }^{1}$, H.-K. Ea ${ }^{1}$, V. Guigonis ${ }^{6} .{ }^{1}$ Rheumatology department, LARIBOISIÉRE Hospital; ${ }^{2}$ Physiology Department, Georges Pompidou European hospital, Paris; ${ }^{3}$ Pharmacy department, Mother and Child hospital, Limoges; ${ }^{4}$ Functional explorations department, TENON Hospital;

${ }^{5}$ Laboratoire Chimie de la Matière Condensée de Paris, UMPC, Paris; ${ }^{6}$ Pediatric Department, Mother and Child hospital, Limoges, France

Background: Tumoral Calcinosis (TC) is a difficult-to-treat complication that can occur during the course of several diseases such as dermatomyositis or genetic hyperphosphatemia. It is a painful and disabling condition that can give rise to local complications including joint mobility reduction, cutaneous ulceration and superinfection. Until now, many treatments have been used with inconstant efficacy.

Objectives: Intravenous sodium thiosulfate gives promising results in calciphylaxis and ectopic calcifications, and intra-lesional injections could be effective for tumoral calcinosis. 
Methods: We report two cases of successful intra-lesional injections of sodium thiosulfate (STS).

Results: Case 1: A 44-years old woman, with history of dermatomyositis developed in 2009 several TC involving the extensor surfaces of the right elbow and forearm, the two ischiatic regions, fingers and lumbar back. As TC kept growing despite successive treatments, including intravenous STS, we proposed weekly intra-lesional injections of $10 \%$ STS in the elbow's lesion. The injected volume varied from 10 to $30 \mathrm{~mL}$ at each session. Fourrier transform infrared spectroscopy (FTIR) of the aspirations confirmed that TC was composed of carbonated-apatite crystals. After 6-month treatment, we observed clinical and radiological regression of elbow TC whereas bottom TC and finger calcifications were unchanged (Fig 1). No side effect was observed except a subcutaneous infection occurring after an injection during the fifth month. This infection resolved with anti-staphycoccal antibiotics. Calcium, bicarbonate and chlorus serum levels, anion gap and eGFR remained unchanged during STS treatment.

Case 2: A 42-year old male presented with a prolonged history of hyperphosphatemic familial TC, confirmed by genetic analysis revealing homozygous mutations in the gene encoding the fibroblast growth factor 23. The extraosseous calcifications comprised a plain lesion on the right side of the left tibia and a massive heterogeneous lesion in the right buttock, making it the sitting position impossible. Treatment with maximal dose of phosphate binders, diet prescription and an attempt to surgically remove of the buttock's lesion were unsuccessful. Topical application of STS led to a near complete disappearance of the tibial lesion but was inefficient to treat the TC at the buttock. Considering the potential efficacy of STS in this patient, local injections of $25 \%$ STS (12 mL every week) were performed. The calcified material aspiration's analysis confirmed that TC was composed of carbonated-apatite crystals. Calcium and bicarbonate serum levels, anion gap and eGFR remained unchanged. After a 12-month treatment of STS injections, the lesion had significantly regressed (Fig 2), allowing the patient to sit again with no pain.
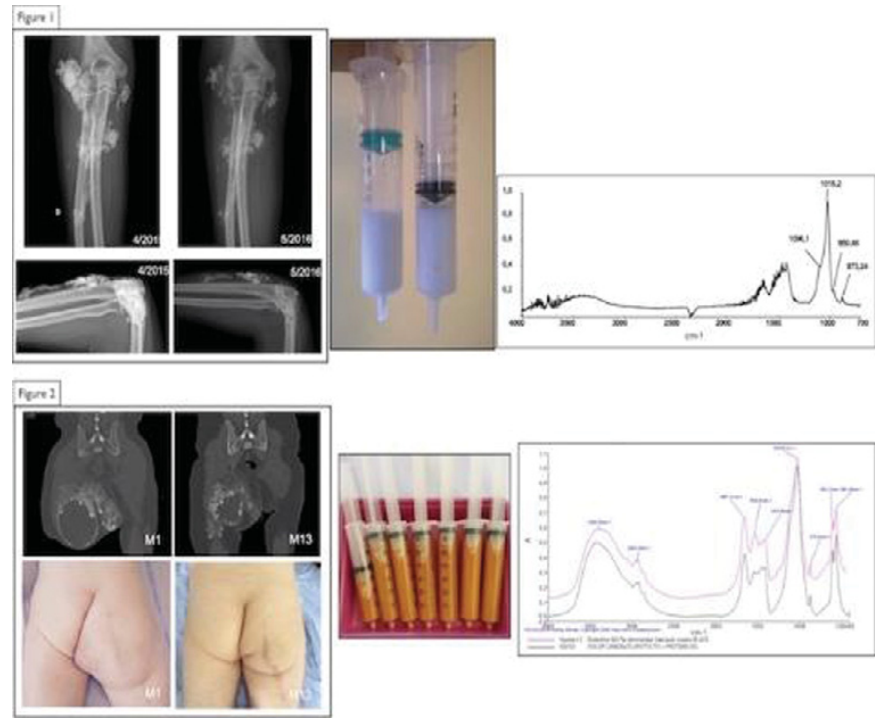

Conclusions: Intra-lesional injection of STS seems to be a promising treatment for TC. More studies are needed to confirm these results, and to understand the mechanisms implicated in the calcinosis resorption.

Disclosure of Interest: None declared

DOI: 10.1136/annrheumdis-2017-eular.2809

\section{AB0883 THE SENSITIVITY OF GOUT SPECIFIC ULTRASOUND SIGNS AT METATARSOPHALANGEAL JOINTS WOULD BE BETTER BY THE DORSAL SURFACE EXAMINATION}

M.A. Mahdi ${ }^{1}$, H. Rkain ${ }^{1}$, M. Erraoui ${ }^{1}$, R. Watfeh ${ }^{2}$, S. Aktaou ${ }^{1}$, L. Tahiri ${ }^{1}$, R. Bahiri ${ }^{1}$, F. Allali ${ }^{1}$, N. Hajjaj-Hassouni ${ }^{1}$. ${ }^{1}$ Rheumatology; ${ }^{2}$ Faculty of Medicine and Pharmacy, University Mohamed V, Rabat, Morocco

Objectives: To compare the prevalence of ultrasonographic gout specific signs at the dorsal and plantar surfaces of the metatarsophalangeal joints (MTP).

Methods: This is a cross-sectional study which includes 15 patients with chronic gout, defined according to the American College of Rheumatology criteria (ACR 1977). Ultrasound (US) examination was performed using a high-frequency linear probe (Toshiba Xario ${ }^{\circledR}$, frequency $(8-14 \mathrm{MHz})$ ) in B mode. 150 articular sites were studied at their dorsal and plantar surfaces. The ultrasound has objectified the presence of two signs: hyperechoic band over the superficial margin of the articular cartilage described as a double contour (DC) and the tophaceous deposits at the joint cavity. We compared the prevalence of the two signs between the dorsal and palmar surfaces at each site studied.

Results: The mean age at onset was $54.7 \pm 12,6$ years, and the median diagnosis duration was $0(0.3)$ years)
The results of the US examination are summarized in Table 1.

Table 1. Prevalence comparison of DC and tophaceous deposits between dorsal and plantar surfaces at MTP joints

\begin{tabular}{lccccccc}
\hline Joints $(\mathrm{N}=150)$ & \multicolumn{3}{c}{ Double contour $(\%)$} & & \multicolumn{3}{c}{ Tophaceous deposits $(\%)$} \\
\cline { 2 - 3 } \cline { 7 - 8 } & Dorsal surface & Plantar surface & $\mathrm{P}$ & & Dorsal surface Plantar surface & $\mathrm{P}$ \\
\hline MTP 1 $(\mathrm{N}=30)$ & 33,3 & 10 & 0,03 & 56,7 & 6,7 & 0,01 \\
MTP 2 $(\mathrm{N}=30)$ & 13,3 & 0 & $<0.001$ & 13,3 & 0 & $<0.001$ \\
MTP 3 $(\mathrm{N}=30)$ & 6,7 & 0 & $<0.001$ & 3,3 & 0 & $<0.001$ \\
MTP 4 $(\mathrm{N}=30)$ & 0 & 0 & $<0.001$ & 6,7 & 0 & $<0.001$ \\
MTP 5 $(\mathrm{N}=30)$ & 3,3 & 0 & $<0.001$ & 10 & 0 & $<0.001$ \\
\hline
\end{tabular}

Conclusions: Our study suggests that globally, DC predilect significatily in dorsal than in plantar surfaces of MTP joints. These results should be verified on a larger population.

Disclosure of Interest: None declared

DOI: 10.1136/annrheumdis-2017-eular.3773

\section{AB0884 PREVALENCE OF ULTRASONOGRAPHIC GOUT SPECIFIC SIGNS OF HAND AND FINGERS JOINTS}

M.A. Mahdi ${ }^{1}$, H. Rkain ${ }^{1}$, M. Erraoui ${ }^{1}$, R. Watfeh ${ }^{2}$, S. Aktaou ${ }^{1}$, L. Tahiri ${ }^{1}$, R. Bahiri ${ }^{1}$, F. Allali ${ }^{1}$, N. Hajjaj-Hassouni ${ }^{1} .{ }^{1}$ Rheumatology; ${ }^{2}$ Faculty of Medicine and Pharmacy, University Mohamed V, Rabat, Morocco

Objectives: To evaluate the prevalence of ultrasonographic gout specific signs of hand and fingers joints.

Methods: This is a cross-sectional study which includes 15 patients with chronic gout, defined according to the American College of Rheumatology criteria (ACR 1977). Ultrasound (US) examination was performed using a high-frequency linear probe (Toshiba Xario ${ }^{\circledR}$, frequency $(8-14 \mathrm{MHz})$ ) in B mode. 540 articular sites were studied at their dorsal surface. The ultrasound has objectified the presence of two signs: hyperechoic band over the superficial margin of the articular cartilage described as a double contour (DC) and the tophaceous deposits at the joint cavity.

Results: The mean age at onset was $54.7 \pm 12,6$ years, and the median diagnosis duration was 0 (0.3) years)

The results of the US examination are summarized in Table 1.

Table 1. Prevalence of ultrasonographic gout specific signs of the wrist, MCP, PIP and DIP joints in the studied population

\begin{tabular}{lcc}
\hline Joints $(\mathrm{N}=540)$ & Double contour $(\%)$ & Tophaceous deposits $(\%)$ \\
\hline Wrist joints $(N=120)$ & 12,6 & 36 \\
Radiocarpal $(\mathrm{N}=30)$ & 20 & 43,3 \\
Ulnocarpal $(\mathrm{N}=30)$ & 13,3 & 50 \\
Scaphotrapezial $(\mathrm{N}=30)$ & 3,3 & 16,7 \\
Trapeziometacarpal $(\mathrm{N}=30$ & 13,3 & 43,3 \\
MCP $(\mathrm{N}=150)$ & 8 & 18 \\
MCP 1 $(\mathrm{N}=30)$ & 3,3 & 16,7 \\
MCP 2 $(\mathrm{N}=30)$ & 13,3 & 33,3 \\
MCP 3 $(\mathrm{N}=30)$ & 6,7 & 16,7 \\
MCP 4 $(\mathrm{N}=30)$ & 6,7 & 3,3 \\
MCP 5 $(\mathrm{N}=30)$ & 10 & 20 \\
PIP $(N=150)$ & 4 & 16,6 \\
IP $(\mathrm{N}=30)$ & 3,3 & 30 \\
PIP 2 $(\mathrm{N}=30)$ & 6,7 & 16,7 \\
PIP 3 $(\mathrm{N}=30)$ & 6,7 & 20 \\
PIP 4 $(\mathrm{N}=30)$ & 3,3 & 10 \\
PIP 5 $(\mathrm{N}=30)$ & 0 & 6,7 \\
DIP $(N=120)$ & 0,8 & 10 \\
DIP 2 $(\mathrm{N}=30)$ & 0 & 13,3 \\
DIP 3 $(\mathrm{N}=30)$ & 3,3 & 13,3 \\
DIP 4 $(\mathrm{N}=30)$ & 0 & 6,7 \\
DIP 5 $(\mathrm{N}=30)$ & 0 & 6,7
\end{tabular}

Conclusions: This study showed a predilection for the gout specific ultrasound signs (DC and tophaceous deposits) of the wrist and MCP joints. The contribution of musculoskeletal ultrasound seems to be very interesting to objectify the presence of gout specific signs in the hand and fingers joints.

Disclosure of Interest: None declared

DOI: 10.1136/annrheumdis-2017-eular.3877

\section{AB0885 PREVALENCE OF ULTRASONOGRAPHIC GOUT SPECIFIC SIGNS OF FOOT JOINTS}

M.A. Mahdi ${ }^{1}$, H. Rkain ${ }^{1}$, M. Erraoui ${ }^{1}$, R. Watfeh ${ }^{2}$, S. Aktaou ${ }^{1}$, L. Tahiri ${ }^{1}$, R. Bahiri ${ }^{1}$, F. Allali ${ }^{1}$, N. Hajjaj-Hassouni ${ }^{1}$. ${ }^{1}$ Rheumatology; ${ }^{2}$ Faculty of Medicine and Pharmacy, University Mohamed V, Rabat, Morocco

Objectives: To evaluate the prevalence of ultrasonographic gout specific signs of foot joints.

Methods: This is a cross-sectional study which includes 15 patients with chronic gout, defined according to the American College of Rheumatology criteria (ACR 1977). Ultrasound (US) examination was performed using a high-frequency linear probe (Toshiba Xario ${ }^{\circledR}$, frequency $(8-14 \mathrm{MHz})$ ) in B mode. 330 articular sites were 